\title{
Periodic Quasiregular Mappings of Finite Order
}

David Drasin and Swati Sastry

\begin{abstract}
The authors construct a periodic quasiregular function of any finite order $\rho, 1 \leq \rho<\infty$. This completes earlier work of O. Martio and U. Srebro.
\end{abstract}

\section{Introduction}

Let $f$ be a (sense-preserving) quasiregular map on $\mathbb{R}^{m}(m \geq 2)$. Thus $f$ is $A C L^{m}$ and there is a $K<\infty$ with

$$
\left|f^{\prime}(x)\right|^{m} \leq K J_{f}(x) \text { a.e., }
$$

where the left side is the norm of the induced operator on the tangent space at $x$, and the right side is the Jacobian determinant. The now-standard reference is Rickman's monograph [4]. These mappings carry much of the geometric theory of analytic and meromorphic functions to higher dimensions. Suppose in addition that $f$ is entire. We then set

$$
M(r, f)=\max _{|x| \leq r}|f(x)|,
$$

and define the order $\rho$ of $f$ by

$$
\rho=\limsup _{r \rightarrow \infty} \frac{\log \log M(r, f)}{\log r} .
$$

Perhaps the most important function in the theory is V. Zoric's analogue of the exponential function, $Z(x)$ (cf. [4, p. 15]). It it is not a local homeomorphism, has order one, and is periodic in $m-1$ of the variables. Using the Zoric function, O. Martio and U. Srebro [3] observed that there exist $(m-1)$-periodic mappings of order 1 and $\infty$, and (Theorem 8.7) that 1 is a lower bound for the orders of such functions.

2000 Mathematics Subject Classification: Primary, 30C65; Secondary: 30C85, 30D99. Keywords: Zoric function, power mapping. 
They raise a question [3, p. 38] which is answered by our

Theorem 1.1 Let $\rho, 1 \leq \rho \leq \infty$ be given. Then there exists an $(m-1)$ periodic $K(m)$-quasiregular map $g$ of exact order $\rho$.

In view of [3], this theorem has significance only when $\rho \in(1, \infty)$. The main step in our construction is Theorem 2.1, in which we associate an entire $K-\mathrm{qr}$ map $f$ to any of a class of slowly increasing functions $\nu(r)$ which satisfy $(2.2)$ below; $K$ will be independent of the specific choice of $\nu$ and depend only on the dimension $m$. For example, let $\nu(r)=\rho(\log r)^{\rho-1}$ for any fixed $\rho>1$. Not only will we have $\log M(r, f) \sim(\log r)^{\rho}$, but for most large $x$,

$$
\log |f(x)| \sim(\log |x|)^{\rho},
$$

where the symbol $\sim$ means that the ratio of the two sides is bounded above and below by positive constants. From this it is routine to see that

$$
g(x)=f \circ Z(x)
$$

is entire, $(m-1)$-periodic, $K_{1}-\mathrm{qr}$ and of exact order $\rho$. In the special case $m=2$ and $K=1$ (analytic functions), the functions of Theorem 2.1 exhaust the class of entire functions of very slow completely regular growth. These functions are discussed, for example, in $[1, \S 6.7]$.

In [3, p. 38] Martio and Srebro raise another question, for which Theorem 1.1 yields a negative answer. So long as $\rho>1$, the function $f$ will have infinitely many zeros in $\mathbb{R}^{m}$. Then (1.3) guarantees that $g$ also has infinitely many zeros in each fundamental region $\Omega$ of the function $Z$ in $\mathbb{R}^{m}$. Martio and Srebro had asked if $\rho$ must always be infinite whenever $g$ is quasiregular, $(m-1)$-periodic and some equation $g(x)=a$ has infinitely many solutions in a fundamental region. They show in Theorem 8.7 that when $\rho=1$ each $a \in \mathbb{R}^{m}$ has only finitely many preimages in each $\Omega$. Our Theorem $1.1 \mathrm{im}-$ plies that their theorem is sharp: when $f$ is chosen as in (1.2) and (1.3), then $g$ assumes all values infinitely often in each $\Omega$.

\section{A generalization of the power mapping}

Theorem 2.1 Let $\nu(r)$ be a positive increasing function such that $\nu \rightarrow \infty$,

$$
r \nu^{\prime}(r)<\frac{\nu(r)}{2}, \quad r \nu^{\prime}(r)=o(\nu(r)) \quad(r \rightarrow \infty)
$$

and set

$$
A(r)=\exp \int_{1}^{r} \nu(t) t^{-1} d t
$$


Then there exists an entire $K=K(m)$ - qr map $f$ on $\mathbb{R}^{m}$ with

$$
M(r, f) \sim A(r) \quad(r \rightarrow \infty) .
$$

Moreover, on $S(r)=\{x ;|x|=r\}$, we have $\left(h_{m-1}\right.$ is $(m-1)$-Hausdorff measure)

$$
|f(x)|>(1+o(1)) A(r) \quad(|x| \rightarrow \infty, x \in S(r) \backslash E(r)),
$$

where $h_{m-1}(E(r))=o\left(r^{m-1}\right)=o\left(h_{m-1}(S(r))\right)$.

When $\nu(r) \equiv n \in \mathbb{Z}^{+}$, the construction is a more complicated version of the power mapping as described in [4, Ch.1, §3.2]. The theorem can be reformulated to allow $\nu$ to tend to a finite limit, but since $\nu \rightarrow \infty$ in cases of interest, we impose this additional hypothesis.

The map $f$ depends on a sequence $\left\{r_{n}\right\}$ with

$$
\nu\left(r_{n}\right)=n,
$$

and will be defined on the boundary of each $m$-cube $Q_{r}$,

$$
Q_{r}=\left\{x ;\|x\|_{\infty} \leq r\right\} .
$$

Every $\partial Q_{r}$ has $2 m$ faces $\left\{F_{j}\right\}$, on each of which $x_{j} \equiv \pm r$ for some $1 \leq j \leq m$.

Note from (2.2) and (2.5) that

$$
n \log \frac{r_{n+1}}{r_{n}} \rightarrow \infty
$$

since $1=\int_{r_{n}}^{r_{n+1}} t \nu^{\prime}(t) d t / t=o(1) n \log \left(r_{n+1} / r_{n}\right)$. We choose $\varepsilon_{0}=\varepsilon_{0}(m)$ with

$$
0<\varepsilon_{0}<\frac{1}{2}, \quad \sin ^{-1} \varepsilon_{0}<\frac{1}{2} \sin ^{-1} m^{-1 / 2} .
$$

Then (2.6) yields $r_{0}$ and $n_{0}=n_{0}\left(\varepsilon_{0}, \nu\right) \geq 4$ so that

$$
\begin{gathered}
(m+1) r \nu^{\prime}(r) / \nu(r) \leq \varepsilon_{0} \quad\left(r>r_{0}\right), \quad \nu\left(r_{0}\right)=n_{0} \in \mathbb{Z}, \\
n \log \frac{r_{n+1}}{r_{n}}>(m+1) \varepsilon_{0}^{-1} \quad\left(n \geq n_{0}\right) .
\end{gathered}
$$

In this and the next two sections we construct $f$ on $\cup \partial Q_{r}\left(r \geq r_{0}\right)$, leaving the simpler range $0 \leq r \leq r_{0}$ to $\S 5$.

With the $\left\{r_{n}\right\}$ as in $(2.5)$, let $J_{n}\left(n \geq n_{0}\right)=\left[r_{n}, r_{n+1}\right]$. We partition $J_{n}$ into $m+1$ intervals $J_{n}^{\ell}=\left[r_{n, \ell}^{\prime}, r_{n, \ell}^{\prime \prime}\right](0 \leq \ell \leq m)$, subject to $r_{n, 0}^{\prime}=r_{n}, r_{n, \ell}^{\prime \prime}=$ $r_{n, \ell+1}^{\prime}, r_{n, m}^{\prime \prime}=r_{n+1} ;(2.9)$ shows that we may suppose

$$
\varepsilon_{0} \log \left(\frac{r_{n, \ell}^{\prime \prime}}{r_{n, \ell}^{\prime}}\right)=\log \left(\frac{n+1}{n}\right),\left(1 \leq \ell \leq m, n \geq n_{0}\right) .
$$


Thus for each $1 \leq \ell \leq m, r_{n, \ell}^{\prime \prime}=(1+o(1)) r_{n, \ell}^{\prime}(n \rightarrow \infty)$, while $r_{n, 1}^{\prime} / r_{n} \rightarrow \infty$. Since $n \geq n_{0}$ is usually fixed in $\S \S 2-4$, we often ignore it in our notations.

In $\S 3$ we construct $f$ on

$$
\bigcup_{n \geq n_{0}} \bigcup_{r \in J_{n}^{0}} Q_{r}
$$

where we set $J^{0}=J_{n}^{0}=\left[r_{n, 0}^{\prime}, r_{n, 0}^{\prime \prime}\right] \equiv\left[r_{0}^{\prime}, r_{0}^{\prime \prime}\right] n \geq n_{0}$. The situation is simpler here since the combinatorics on each $\partial Q_{r}$ does not change with $r$, while in $\S 4$ we modify this approach on the $\left\{J_{n}^{k}\right\}, n \geq n_{0}, k \geq 1$.

The map $f$ has to evolve in $J=J_{n}$ subject to:

(A) on $\partial Q_{r_{n}} f$ is (a constant multiple of) a power-type map of 'degree' $n$ (cf. [4, p. 14]). Thus each of the $2 m$ faces of $\partial Q_{r_{n}}$ is first divided into $(2 n)^{m-1}$ congruent $(m-1)$-'boxes' $\mathcal{K}$, where a box is the product of $m$ closed intervals: $\mathcal{K}=I_{1} \times \ldots \times I_{m}$, with one $I_{j}=\{+r\}$ or $\{-r\}$ and $\left|I_{i}\right|=r / n$ when $i \neq j$. With $S_{m-1}=2^{m-1}(m-1)$ ! as determined below (3.1), we then divide each $\mathcal{K}$ into $S_{m-1}(m-1)$-simplices $\Lambda_{r}$. The map $f$ is defined on each $\Lambda_{r}$ by (3.6), so that $f$ is $K$-qc on $\Lambda_{r}, K$-qr on $Q_{r}$, with $|f(x)| \sim A\left(r_{n}\right)$ for $x \in \partial Q_{r_{n}}$;

(B) situation (A) holds on $\partial Q_{r_{n+1}}$, with $n+1$ in place of $n$;

(C) the process is such that $f$ is $K$-qr and $|f(x)| \sim A(|x|)$ for most $x$ on every $\partial Q_{r}, r \geq r_{0}$.

We conclude this section with a $P L$ version of the sphere $S^{m}$. While Rickman's map is based on the manifold $S^{m}$ being in the range (and is a so-called Alexander map) our construction in $\S 4$ seems to require the polyhedron $P$ of Proposition 2.12. Let $S^{\prime}=\left\{\left|x^{\prime}\right|=1\right\} \cap\left\{x_{m}=0\right\}$ be the unit $(m-2)$-sphere. Depending on the context, we may view $\alpha \in S^{\prime}$ as a vector in $\mathbb{R}^{m-1}$ or one in $\mathbb{R}^{m}$ whose final coordinate is zero. Choose $m$ points $\alpha^{0}, \ldots, \alpha^{m-1} \in S^{\prime}$ so that the vectors $\alpha^{j}-\alpha^{0}(1 \leq j \leq m-1)$ form a basis of $\mathbb{R}^{m-1}$ which is $L(m)$-bilipschitz equivalent to the standard basis, the origin is in the convex hull of the $\left\{\alpha^{i}\right\}$, and the map $\left(\alpha^{j}-\alpha^{0}\right) \rightarrow e^{j}$ is sensepreserving; the $\left\{e^{j}\right\}$ are the standard basis of $\mathbb{R}^{m-1}$. Let $\Delta$ be the convex hull of the $\left\{\alpha^{i}\right\}$, and $s \Delta=\{s p ; p \in \Delta\}$. For $s>0$ and $q=s \sum \lambda_{i} \alpha^{i} \in \Delta_{s}$, consider the function

$$
\lambda(q)=\lambda_{s}(q)=m s \inf _{i} \lambda_{i} \quad\left(q \in \Delta_{s}\right) .
$$

(The factor $m$ ensures that $\max _{\Delta_{s}} \lambda(q)=s$ ). 
Proposition 2.12 For each $s>0$, the graph of the function $\lambda_{s}(q), q \in \Delta_{s}$, is a polyhedron $P^{+}=P_{s}^{+} \subset\left\{x_{m} \geq 0\right\}$. If we define $P^{-}$as the graph of $-\lambda_{s}(q)$, then

$$
P=P^{+} \cup P^{-}
$$

is a polyhedron composed of subsets of a finite number of hyperplanes with 0 in its interior. If $q \in \partial \Delta_{s}$, then $\lambda(q)=0$.

The ray from 0 to the point $(q, \pm \lambda(q)) \in P$ makes an angle $\Phi$ with $P$ such that

$$
|\sin \Phi|>3 \tau>0
$$

where $\tau$ depends only on the specific choice of the $\left\{\alpha^{i}\right\}$.

Proof. It suffices to consider $s=1$. Then $P$ determined by $2 m$ hyperplanes each of which contains $m-1$ of the $\left\{\alpha^{i}\right\}$ and one of the points $(\alpha, \pm 1)$, where $\alpha=\sum \alpha^{i} / m$ is the barycenter of $\Delta$, so it is clear that 0 is interior to $P$. The normal to each of these hyperplanes has a nonzero component orthogonal to the hyperplane $\left\{x_{m}=0\right\}$, so the result follows by elementary linear algebra.

\section{The first stage}

Recall the $\left\{J_{n}\right\}=\left\{\cup_{0 \leq \ell \leq m} J_{n}^{\ell}\right\}, n \geq n_{0}$, from the discussion of (2.10). Let $r \in J_{n}^{0}$, and consider a face $F \subset \partial Q_{r}$ on which $x_{j}=\epsilon r$, for $\epsilon= \pm 1$. Then for $1 \leq i \leq n, i \neq j$, the planes

$$
\Pi_{p}^{i}(n)=\left\{x_{i}=p r / n\right\}, \quad|p| \leq n,
$$

divide $F$ into $(2 n)^{m-1}(m-1)$-boxes $\mathcal{K}$, and barycentric subdivision of each box in turn partitions $F$ into a union of $(m-1)$-simplices $\Lambda_{r}$, which are positively or negatively oriented with respect to the standard orientation $\partial Q_{r}$ inherits from $\mathbb{R}^{m}$. As $r \in \cup_{n \geq n_{0}} J_{n}^{0}$ and $1 \leq j \leq m$ vary, note that each vertex $b(r)$ of $\Lambda_{r}$ may be associated to a vector $p \in \mathbb{Z}^{m}$ :

$$
b(r)=\left(\frac{p_{1}}{2 n}, \frac{p_{2}}{2 n}, \ldots, \frac{p_{m}}{2 n}\right) r
$$

with $\left|p_{i}\right| \leq 2 n$; on $F, p_{j} \equiv 2 \epsilon n$. Each $\Lambda_{r}$ is $L$-bilipschitz equivalent to the standard $(m-1)$-simplex, up to the scaling factor (cf. (2.3))

$$
\frac{r}{\nu(r)}=\frac{A(r)}{A^{\prime}(r)}
$$


with $L=L(m)$. Thus

$$
L^{-1} \frac{r}{\nu(r)} \leq\left|b^{i}(r)-b^{j}(r)\right| \leq L \frac{r}{\nu(r)} \quad(i \neq j)
$$

The vertices of $\cup_{\partial Q_{r}} \Lambda_{r}$ are put into $m$ classes $b^{i}, 0 \leq i \leq m-1$, using the standard model $\Delta$ of Proposition 2.12. On some face $F \subset \partial Q_{r}$ choose a positively oriented simplex $\Lambda_{r}^{0}$, and label its vertices $b^{i}(r), 0 \leq i \leq m-1$, the ordering taken so that the map

$$
\sum \lambda_{i} b^{i}(r) \rightarrow \sum \lambda_{i} \alpha^{i} \quad\left(\lambda_{1} \geq 0, \sum \lambda_{i}=1\right)
$$

from $\Lambda_{r}^{0}$ to $\Delta$ has positive Jacobian. We may then consistently assign clases $b^{i}$ to any of the vertices of all $\Lambda_{r} \subset \partial Q_{r}$, so that if $\Lambda_{r}$ and $\Lambda_{r}^{\prime}$ share a lower dimensional subsimplex, the vertices common to both simplexes belong to the same class. Note that the mapping (3.4) when defined on each simplex $\Lambda_{r}$ is sense preserving if $\Lambda_{r}$ is positively oriented, and sense reversing otherwise.

With $s=A(r)\left(r \in J_{n}^{0}\right)$ from (2.3), let $p=\sum \lambda_{i} b^{i}(r) \in \Lambda_{r} \subset \partial Q_{r}$, set

$$
p^{\prime}=s\left(\sum \lambda_{i} \alpha^{i}\right) \quad(s=A(r))
$$

and, recalling the function $\lambda\left(p^{\prime}\right)$ of $(2.11)$, define

$$
f(p)=\left(p^{\prime}, \pm \lambda\left(p^{\prime}\right)\right)=\left(s \sum \lambda_{i} \alpha^{i}, \pm \lambda\left(p^{\prime}\right)\right) \quad(s=A(r))
$$

The first entry on the right side of (3.6) is an $(m-1)$-vector, and the second is a scalar, and the \pm sign is taken according to whether (3.4) preserves or reverses orientation. Thus (3.6) is always sense preserving.

Lemma 3.7 Let $\mathcal{B}: e^{1}, \ldots, e^{m}$ be the standard basis of $\mathbb{R}^{m}$. Then there is a $K_{1}<\infty$ such that at almost each point $p$ and $f(p)$ exist bases $\mathcal{V}=\left\{v^{i}\right\}$ and $\mathcal{W}=\left\{w^{i}\right\}$ of the tangent spaces $T_{p}$ and $T_{f(p)}$ such that the linear maps determined by

$$
e^{i} \leftrightarrow v^{i}, \quad e^{i} \leftrightarrow w^{i}
$$

are $K_{1}$-quasiconformal. Moreover, if $\mathcal{J}_{f}$ is the Jacobian matrix relative to the bases $\mathcal{V}$ and $\mathcal{W}$, then

$$
\mathcal{J}_{f}=A^{\prime}(r) I
$$

Hence, if $K_{2}$ is the dilatation of the map (3.4), then $f$ is $K=K_{1}^{2} K_{2}$ quasiregular. 
Proof. Given $p=\sum \lambda_{i} b^{i}(r) \in \Lambda_{r} \subset \partial Q_{r}$, define $p^{\prime}$ by (3.5). Assume there is a $+\operatorname{sign}$ in (3.6), and $\lambda_{k}=\min _{i} \lambda_{i}$ in a neighborhood of $p$. The basis for $T_{p}$ consists of $\mathcal{V}=\left\{v^{1}, \ldots, v^{m}\right\}$ such that $v^{m}=\sum \lambda_{i}\left(b^{i}\right)^{\prime}(r)$, and for $1 \leq t \leq m-1$, the $\left\{v^{t}\right\}$ are the vectors $(\nu(r) / r)\left(b^{\sigma(t)}-b^{k}\right)$, where the $\{\sigma(t)\}_{i=1}^{m-1}$ exhaust the range $1 \leq t \leq m, \sigma \neq k$, ordered so that $\mathcal{V}$ is positively oriented with respect to $\mathcal{B}$. At $f(p)=\left(p^{\prime}, \lambda(p)\right)$ the basis of $T_{f(p)}$ will be normalized $D f$-images of $\mathcal{V}$, so that when $t<m, w^{t}=$ $\left(\alpha^{h(t)}-\alpha^{k},-m\right)$. When $r \in J_{n}^{0}\left(n \geq n_{0}\right)$ the final basis vector $w^{m}$ in $\mathcal{W}$ is $w^{m}=\left(\sum \lambda_{i} \alpha^{i}, m \lambda_{k}\right)$, but this will be modified in Lemma 4.7 for the situation $r \in \cup_{\ell \geq 1} J_{n}^{\ell}, n \geq n_{0}$.

Since $\lambda\left(p^{\prime}\right)$ is also determined by the coefficient $\lambda_{k}$ of $b^{k}$ for $p^{\prime}$ near $p,(3.6)$ shows that $f$ is linear near $p$. Hence if $t<m$ and $h$ is small,

$$
p+h v^{t}=b^{k}+\sum_{i \neq \sigma(t), k} \lambda_{i} b^{i}+\left(\lambda_{\sigma(t)}+h(\nu(r) / r)\right)\left(b^{\sigma(t)}-b^{k}\right),
$$

and (2.3), (2.11), (3.5) and (3.6) yield for $1 \leq t \leq m-1$ that

$$
D f\left(v^{t}\right)=\frac{f\left(p+h v^{\sigma(t)}\right)-f(p)}{h}=\frac{\nu(r)}{r} A(r)\left(\alpha^{\sigma(t)}-\alpha^{k},-m\right) \equiv A^{\prime}(r) w^{t} .
$$

Next, consider $D f\left(v^{m}\right)$. Let $r^{\prime}=r+h$ and consider the image of $p+h v^{m}=$ $\sum \lambda_{i}\left(b^{i}+h\left(b^{i}\right)^{\prime}\right)$. By $(3.1)$,

$$
p+h v^{m}=\sum \lambda_{i}\left(b^{i}(r)+h\left(b^{i}\right)^{\prime}(r)\right)=\sum \lambda_{i} b^{i}\left(r^{\prime}\right) \quad\left(r^{\prime}=r+h\right),
$$

so that $f\left(p+h v^{m}\right)-f(p)=\left(A\left(r^{\prime}\right)-A(r)\right)\left(\sum \lambda_{i} \alpha^{i}, m \lambda_{k}\right)$, and

$$
D f\left(v^{m}\right)=A\left(r^{\prime}\right) w^{m} \text {. }
$$

We check that the bases $\mathcal{V}$ and $\mathcal{W}$ satisfy the assertions of Lemma 3.7. First consider $p \in \Lambda_{r}$. The explicit form of the simplices $\Lambda_{r}$ and the arrangement of the $\{\sigma(t)\}$ show that the first $m-1$ vectors $v^{i}$ form part of such a basis at $T_{p}$ and lie parallel to that face $F$ of $\partial Q_{r}$ which contains $p$, while (3.3) implies $\left|v^{i}\right| \sim 1$. In addition, we deduce from (3.1) that $\left|v^{m}\right| \sim 1$, and that (the vector from 0 to) $p$ makes an angle $\Theta$ with $F$ such that $|\sin \Theta|>m^{-1 / 2}$, so $\Theta$ is uniformly bounded away from 0 . Thus $\mathcal{V}$ is related to $\mathcal{B}$ as claimed in the Lemma.

Now consider $\mathcal{W}$. That $\left|w^{i}\right|=\left|\left(\alpha^{i}-\alpha^{k},-m\right)\right| \sim 1$ for $i<m$ follows from properties of the $\left\{\alpha^{i}\right\}$. In addition, we have that $\left|w^{m}\right|=\left|\left(\sum \lambda_{i} \alpha^{i}, m \lambda_{k}\right)\right| \sim 1$. This follows from (2.11) and (3.6) when $\lambda_{k}\left(=\min \lambda_{i}\right)>\eta>0$, but when $\lambda_{k}$ is small, then $\sum \lambda_{i} \alpha^{i}$ lies near $\partial \Delta$, and so $\sum \lambda_{i}$ already has magnitude at least $h$ for some fixed $h>0$. To check that the $\left\{w^{i}\right\}$ span $\mathbb{R}^{m}$ appropriately, note that the $\left\{w^{j}\right\}(j<m)$ span the tangent plane at $f(p) \in A(r) P$. Hence (2.13) ensures that $w^{m}$ has a uniformly nontrivial normal component to $A(r) P$ at $f(p)$. 


\section{Interpolation}

In order to define $f$ on $\partial Q_{r}$ for $r \in J_{n}^{k}\left(k \geq 1, n \geq n_{0}\right)$ we follow the scheme of $\S 3$, but need to arrange new simplices (or partial simplices) so that (B) in $\S 2$ holds when $r=r_{n+1}$. We do this by working with the $(m-1)$ free coordinates on a given face $F$ one at a time, and when $r \in J_{n}^{\ell}$, this will be $x_{\ell}$.

Consider, for example, the face $F \subset \partial Q_{r}$ on which $x_{j} \equiv r$. For each $1 \leq i \leq m, i \neq j, F$ again is partitioned by $(m-1)$-planes orthogonal to the $x_{i}$-axis. This has already been described when $r \in J^{0}$, so consider a fixed $\ell \geq 1$. Then for each $i<\ell, i \neq j$, the planes

$$
\Pi_{p}^{i}(n+1)=\left\{x_{i}=\operatorname{pr} /(n+1)\right\}, \quad|p| \leq n+1
$$

divide $F$ into $2(n+1)$ congruent slices, and when $i>\ell, i \neq j$, the $\left\{\Pi_{p}^{i}(n)\right\}$, $|p| \leq n$ of (3.1) divide $F$ into $2 n$ congruent slices.

We next consider $i=\ell$, and recall $\varepsilon_{0}$ in (2.7) and that $J_{n}^{\ell}=\left[r_{\ell}^{\prime}, r_{\ell}^{\prime \prime}\right]$. Then use (2.10) to define $\nu_{\ell}(r)$ with

$$
\begin{aligned}
\nu_{\ell}\left(r_{\ell}^{\prime}\right) & =n, \nu_{\ell}\left(r_{\ell}^{\prime \prime}\right)=n+1, \\
\frac{d\left(\log \nu_{\ell}(r)\right)}{d(\log r)} & \equiv \frac{r \nu_{\ell}^{\prime}(r)}{\nu_{\ell}(r)}=\frac{1}{\log \left(r_{\ell}^{\prime \prime} / r_{\ell}^{\prime}\right)} \equiv \varepsilon_{0} \quad\left(r_{\ell}^{\prime} \leq r \leq r_{\ell}^{\prime \prime}\right),
\end{aligned}
$$

and partition $F$ by planes $\Pi_{p}^{\ell}\left(\nu_{\ell}\right) \equiv\left\{x_{\ell}=p r / \nu_{\ell}(r), p \in \mathbb{Z}, 0 \leq|p| \leq n\right\}$. As $r$ increases in $J_{n}^{\ell}$, each $\Pi_{ \pm p}^{\ell}\left(\nu_{\ell}\right)$ recedes from $\left\{x_{\ell}= \pm r\right\}$ and so for the appropriate choice of $n^{*} \in\{n, n+1\}$, the $\left\{\Pi_{p}^{i}\left(n^{*}\right)\right\}\left(i \neq j, \ell\right.$, and $\left.|p| \leq n^{*}\right)$, $\left\{\Pi_{p}^{\ell}\left(\nu_{\ell}\right)\right\}$ and $\left\{x_{\ell}= \pm r\right\}$ create new boxes $\mathcal{K} \subset F$, which when $r=r_{\ell}^{\prime \prime}$ are all congruent. Boxes whose boundary is disjoint from $\left\{x_{\ell}= \pm r\right\}$ are called interior boxes, and the others are boundary boxes.

As in $\S 3$, these boxes must be divided into simplices, and $f$ defined simplex by simplex. If $\mathcal{K}_{0}$ is an interior box, its barycentric subdivision leads at once to oriented simplies $\Lambda_{r}$ as in $\S 3$, with vertices $b(r)$ having coordinates $b_{i}(r)$, such that for $i \neq j, i<\ell$, we have $b_{i}=\left(2 p_{i}\right) r / 2(n+1)\left(\left|p_{i}\right| \leq n+1\right)$, while $b_{\ell}=\left(2 p_{\ell}\right) r /\left(2 \nu_{\ell}(r)\right) \quad\left(\left|p_{\ell}\right| \leq n\right)$ and $b_{i}=\left(2 p_{i}\right) r /(2 n),\left|p_{i}\right| \leq n$ when $i>\ell, i \neq j$. On $F$ we have $b_{j} \equiv r$. This again allows the simplex structure and orientation to be transferred to the interior boxes. The only new feature is that the coordinate $b_{\ell}$ of each vertex satisfies

$$
r b_{\ell}^{\prime}=b_{\ell}\left(1-\frac{r \nu_{l}^{\prime}}{\nu_{\ell}}\right) \equiv b_{\ell}\left(1-\varepsilon_{0}\right)
$$

instead of what appears in (3.2). Since $n \leq \nu_{\ell}(r) \leq n+1$, these simplices $\Lambda_{r}$ are $\left(1+o(1)\right.$-bilipschitz equivalent to those $\Lambda_{r}$ for $r \in J_{n}^{0}$, and so the mappings (3.4) are uniformly $(1+o(1)) K_{2}$-qc (perhaps sense reversing). 
We next consider the boundary boxes, and partition them into what we call partial simplices $\Lambda_{r}^{*}$. It suffices to work in $\left\{x_{\ell} \geq 0\right\} \cap Q_{r}$. The $x_{i}$-coordinates $(i \neq \ell)$ of these boxes are the same as those corresponding to vertices

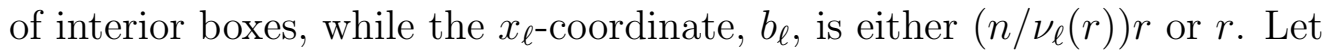

$$
r^{*}=\frac{1}{2}\left(1+\frac{n}{\nu_{\ell}(r)}\right) r=\left(\frac{n+\nu_{\ell}(r)}{2 \nu_{\ell}(r)}\right) r
$$

and $H:\left\{x_{\ell}=r^{*}\right\}$. Then $H$ lies midway between $\Pi_{n}^{\ell}\left(\nu_{\ell}\right)$ and $\left\{x_{\ell}=r\right\}$, and each boundary box $\mathcal{K}$ is divided by $H$ into two congruent subboxes $\mathcal{K}_{ \pm}$. Let $\mathcal{K}_{-}=\mathcal{K} \cap\left\{\left(n r / \nu_{\ell}\right) \leq x_{\ell} \leq r^{*}\right\}$ and $\mathcal{K}_{+}$the reflection of $\mathcal{K}_{-}$in $H$. In an obvious sense $\mathcal{K}_{-}$may be considered as a subset of a (phantom) box $\mathcal{K}^{\prime}$ which is bounded by the hyperplanes $\Pi_{n}^{\ell}\left(\nu_{\ell}\right)$ and $\Pi_{n+1}^{\ell}\left(\nu_{\ell}\right) \equiv\left\{x_{\ell}=\right.$ $\left.r(n+1) / \nu_{\ell}(r)\right\}$, as well as the various hyperplanes $\Pi_{p}^{i}\left(n^{*}\right)\left(i \neq j, \ell, n^{*} \in\right.$ $\{n, n+1\})$ which meet $\partial \mathcal{K}$. In particular, $\mathcal{K}_{-}^{\prime}$ may be divided into oriented simplices $\Lambda_{r}$ generated by vertices in the classes $b^{i}(r)$ exactly as with the interior boxes $\mathcal{K}$. The vertices $\Lambda_{r}^{*}$ of $\mathcal{K}_{-}$are of the form $\Lambda_{r}^{*}=\Lambda_{r} \cap \mathcal{K}^{\prime}$, with inherited orientation. In the same way, we obtain simplices $\left(\Lambda_{r}^{\prime}\right)^{*} \subset \mathcal{K}_{+}$; these are reflections of the $\left\{\Lambda_{r}^{*}\right\}$ across $H$.

We place $\Lambda_{r}^{*} \subset \mathcal{K}^{\prime}$ in groups according to how many vertices $\Lambda_{r} \supset \Lambda_{r}^{*}$ does not have on $\Pi_{n}^{\ell}\left(\nu_{\ell}\right)$. This number, $t\left(\Lambda_{r}^{*}\right)$, is at least 1 and at most $m-1$. If $\left(\Lambda_{r}^{\prime}\right)^{*} \subset \mathcal{K}_{+}$is the reflection of $\Lambda_{r}^{*}$ across $H$, set $t\left(\Lambda_{r}^{\prime}\right)^{*}=t\left(\Lambda_{r}^{*}\right)$, and note that the vertices of $\Lambda_{r}$ and $\Lambda_{r}^{\prime}$ which contribute to the appropriate $t$ are of the same classes $\left\{b^{i}\right\}$, while orientations of the simplices are reversed. Let $\mathcal{T}=\mathcal{T}\left(\Lambda_{r}^{*}\right)$ be the vertices of $\Lambda_{r}$ which contribute to $t\left(\Lambda_{r}^{*}\right)$ : we call these the phantom vertices.

The mapping $f$ of (3.7) must be modified so that

$$
\begin{aligned}
& f \text { is } L \text {-bilipschitz and } K-q c \text { in each } \Lambda_{r}^{*}, \\
& (f(x))_{m} \geq 0 \text { on } \Lambda_{r}^{*},(f(x))_{m}=0 \text { on } \partial \Lambda_{r}^{*},
\end{aligned}
$$

where $(\cdot)_{m}$ is the $m$-th coordinate. The important requirement is that $(f(x))_{m}$ vanish in $\partial \Lambda_{r}^{*}$; otherwise reflection across the boundary (compare with (3.6)) will not be possible. Note that (3.6) cannot be used, since $(f(x))_{m}$ is usually nonzero when $x \in \mathcal{K}_{+} \cap \mathcal{K}_{-}=H \cap \mathcal{K}$. To avoid this we use $\mathcal{T}$ to modify the function $\lambda$ of (2.11). According to the definition of $t(\Lambda)$, if $p=\sum \lambda_{i} b^{i}(r) \subset \Lambda_{r}^{*}$, then

$$
0 \leq \sum_{\mathcal{T}} \lambda_{i} \leq L(r) \equiv \frac{\nu_{\ell}(r)-n}{2}
$$

where the left equality holds when $p \in \Pi_{n}^{\ell}\left(\nu_{\ell}\right)$ and the right when $p \in H$. 
Thus if $K_{s}$ is the image of $\Lambda_{r}^{*} \cap H$, we have

$$
p^{\prime}=s \sum \lambda_{i} \alpha^{i} \in K_{s} \Longleftrightarrow \sum_{\mathcal{T}} \lambda_{i}=\frac{\nu_{\ell}(r)-n}{2}=L(r) .
$$

Now with $p^{\prime}$ and $\lambda\left(p^{\prime}\right)$ as in (3.5) and (2.11), we define $\lambda_{s}^{*}$ to have the same effect relative to $\Lambda_{r}^{*}$ : if

$$
p^{\prime}=s\left(\sum \lambda_{i} \alpha^{i}\right) \in \Delta_{A(r)}
$$

and $L$ is from (4.4), set

$$
\lambda^{*}\left(p^{\prime}\right)=s \min \left(\lambda\left(p^{\prime}\right),\left(L(r)-\sum_{\mathcal{T}} \lambda_{i}\right)\right),
$$

so that now $\lambda^{*} \equiv 0$ on $K_{A(r)}$. Then when $r \in J_{n}^{\ell}$ and $p \in \Lambda_{r}^{*}(1 \leq \ell \leq m)$, we modify (3.6) to

$$
f(p)=\left(p^{\prime}, \pm \lambda^{*}\left(p^{\prime}\right)\right)=\left(s \sum \lambda_{i} \alpha^{i}, \pm \lambda^{*}\left(p^{\prime}\right)\right) \quad(s=A(r)),
$$

signs chosen so that $f$ is sense preserving. If $p \in \partial \Lambda_{r}^{*}$ and $L(r)-\sum_{\mathcal{T}} \lambda_{i}=0$, then $p \in H$, and the extension to the symmetric $\left(\Lambda_{r}^{\prime}\right)^{*}$ is by reflection across $H$ and $K$.

Lemma 4.7 Let $p \in \partial Q_{r}, r \in J_{n}^{\ell} \ell \geq 1, n \geq n_{0}$. Then at almost every point $p$ there are bases $\mathcal{V}$ and $\mathcal{W}$ of $T_{p}$ and $T_{f(p)}$ so that Lemma 3.7 holds.

Proof. Let $p$ and $p^{\prime}=f(p)$ be as in Lemma 3.7, with $\lambda_{k}$ the minimum $\lambda$ near $p$. Take $\mathcal{V}$ and $\left\{w^{1}, \ldots, w^{m-1}\right\}$ exactly as in Lemma 3.7 , but with the final basis vector, $w^{m}$, replaced by a certain $\hat{w}^{m}$. The first $(m-1)$ components of $\hat{w}^{m}$ are those of $w^{m}$, but $\left(\hat{w}^{m}\right)_{m}$ is modified to the bracketed term in (4.9) below (so that the factor $A^{\prime}(r)$ in (4.9) does not appear in $\hat{w}^{m}$ ).

When $\lambda^{*}\left(p^{\prime}\right)=\lambda\left(p^{\prime}\right)$, the lemma reduces to Lemma 3.7, so we compute $J_{f}$ when in a neighborhood $\Omega$ of $p$

$$
\lambda^{*}\left(p^{\prime}\right)=s\left(L(r)-\sum_{\mathcal{T}} \lambda_{i}\right)<\lambda\left(p^{\prime}\right),
$$

so that the same set $\mathcal{T}$ is common to all $p^{\prime} \in \Omega$. The first $(m-1)$ rows of $J_{f}$ are unchanged, as are all but the diagonal entry of the bottom row. If $p=\sum \lambda_{i} b^{i}(r)$, then $p+h v^{m}=\sum \lambda_{i} b^{i}\left(r^{\prime}\right), r^{\prime}=r+h$, so that once 
again $\sum_{\mathcal{T}} \lambda_{i}$ is invariant. Hence when (4.8) holds, (4.5) and (4.6) show that if $p \in \Omega$ and $h$ is small,

$$
\left(f\left(p+h v^{m}\right)-f(p)\right)_{m}=\left(A\left(r^{\prime}\right)-A(r)\right)\left(L\left(r^{\prime}\right)-\sum_{\mathcal{T}} \lambda_{i}\right)+A(r)\left(L\left(r^{\prime}\right)-L(r)\right),
$$

and hence (2.3), (4.2), (4.4) and (4.6) give that

$$
\begin{aligned}
\left(D f\left(v^{m}\right)\right)_{m} & =A^{\prime}(r)\left(L(r)-\sum_{\mathcal{T}} \lambda_{i}\right)+A(r) \frac{\nu_{k}^{\prime}}{2} \\
& =A^{\prime}(r)\left(L(r)-\sum_{\mathcal{T}} \lambda_{i}\right)+\frac{1}{2}\left(\frac{\nu(r)}{r}\right) A(r)\left(\frac{r \nu_{\ell}^{\prime}}{\nu_{\ell}}\right)\left(\frac{\nu_{\ell}}{\nu}\right) \\
& =A^{\prime}(r)\left[\left(L(r)-\sum \lambda_{i}\right)+\frac{1}{2} \varepsilon_{0}\left(\frac{\nu_{\ell}}{\nu}\right)\right] .
\end{aligned}
$$

Thus if $D f\left(v^{m}\right)=\hat{w}^{m}$, the $m$ th component, $(\hat{w})_{m}$, satisfies

$$
(\hat{w})_{m}=\max \left(\left(w^{m}\right)_{m},\left(L(r)-\sum_{\mathcal{T}} \lambda_{i}\right)+\frac{1}{2} \varepsilon_{0} \frac{\nu_{\ell}}{\nu}\right)
$$

(recall $w^{m}$ from (3.9)). But $\left(1 / 2 \geq\left(L-\sum \lambda_{i}\right) \geq 0\right.$ and $2 \nu \geq \nu_{\ell} \geq(\nu / 2)$ when $r \in J_{n}^{\ell}$. This implies that $1 \geq(\hat{w})_{m} \geq \varepsilon_{0} / 4$.

We check that these bases satisfy the assertions of Lemma 3.7, and so only need consider $\hat{w}^{m}$ in the situation that (4.8) holds near $p$. Now $\varepsilon_{0} / 4 \leq(\hat{w})_{m}$ $\leq\left|w^{m}\right|$, while for $j<m,\left(w^{j}\right)_{m} \equiv-m$. Hence $\hat{w}^{m}$ makes an angle with $\operatorname{span}\left[w^{1}, \ldots, w^{m-1}\right]$ whose sine is uniformly bounded below. This proves the Lemma.

\section{Completion of proof}

To extend $f$ to $Q_{r_{0}}$, recall from $\S 3$ that

$$
f(x)=A\left(r_{0}\right) \Psi(x) \quad\left(x \in \partial Q_{r_{0}}\right)
$$

where $\Psi: \partial Q_{r_{0}} \rightarrow P_{A\left(r_{0}\right)}$, the polyhedron $P$ of Proposition 3.5. Then exactly as in $\left[2\right.$, p. 14] $f$ is extended to the rest of $\mathbb{R}^{m}$ :

$$
f(x)=\left(\frac{r}{r_{0}}\right)^{n_{0}} A\left(r_{0}\right) \Psi\left(\frac{r_{0}}{r} x\right) \quad\left(x \in \partial Q_{r}, r \leq r_{0}\right) .
$$




\section{References}

[1] Hayman, W. K.: Subharmonic Functions, vol. 2. London Mathematical Society Monographs 20. Academic Press, London, 1989.

[2] Martio, O., Rickman, R. and Väisälë, J.: Topological and metric properties of quasiregular mappings. Ann. Acad. Sci. Fenn. Ser. A I 488 (1971), $31 \mathrm{pp}$.

[3] Martio, O. and Srebro, U.: Periodic Quasimeromorphic Mappings in $\mathbb{R}^{n}$. J. Analyse Math. 28 (1975), 20-40.

[4] Rickman, S.: Quasiregular Mappings. Ergebnisse der Mathematik und ihrer Grenzgebiete (3) 26. Springer-Verlag, Berlin, 1993.

Recibido: 28 de febrero de 2000

Revisado: 7 de mayo de 2001

David Drasin

Mathematics Department

Purdue University

West Lafayette, IN 47907, USA

drasin@math . purdue.edu

Swati Sastry

swatisastry@hotmail.com

The authors thank Prof. U. Srebro for raising this problem, and for many helpful comments. The second author also thanks Purdue University for its support during crucial periods of this research. Finally, we acknowledge the referee's persistent demands that the exposition be clearer. 